\title{
Identification and molecular characterization of a novel Babesia orientalis thrombospondin-related anonymous protein (BoTRAP1)
}

Long Yu ${ }^{1,2}$, Qin Liu ${ }^{1,2}$, Xueyan Zhan ${ }^{1,2}$, Yuan Huang ${ }^{1,2}$, Yali Sun ${ }^{1,2}$, Zheng Nie ${ }^{1,2}$, Yangnan Zhao ${ }^{1,2}$, Xiaomeng An ${ }^{1,2}$, Muxiao Li $\mathrm{Li}^{1,2}$, Sen Wang ${ }^{1,2}$, Yangsiqi Ao ${ }^{1,2}$, Cuiqin Huang ${ }^{3,4}$, Lan He $\mathrm{H}^{1,2,5}$ and Junlong Zhao ${ }^{1,2,5^{*}}$

\begin{abstract}
Background: The thrombospondin-related anonymous protein (TRAP) family, a kind of transmembrane protein, is widely distributed with a conserved feature of structure in all apicomplexan parasites and plays a crucial role in the gliding motility and survival of parasites.

Methods: The Babesia orientalis TRAP1 gene (BOTRAP1) was truncated and cloned into a pET-42b expression vector and expressed as a GST-tag fusion protein with a TEV protease site. Rabbit anti-rBoTRAP1 antibody was produced and purified using a protein A chromatography column. Western blot analysis was performed to identify the native protein of BoTRAP1 and differentiate B. orientalis-infected positive from negative serum samples. The localization of BoTRAP1 on merozoites was identified by the indirect florescent antibody test (IFAT).

Results: The partial sequence of the TRAP1 gene was cloned from B. orientalis CDNA and identified to contain a von Willebrand factor A (VWFA) region and a thrombospondin type-1 (TSP-1) domain; it had a length of 762 bp, encoding a polypeptide of 254 amino acid residues with a predicted size of $28.2 \mathrm{kDa}$. The partial sequence was cloned into a pET-42b expression vector and expressed in E. coli as a GST fusion protein. Western blot indicated that rBoTRAP1 has a high immunogenicity and can differentiate B. orientalis-infected positive and negative serum samples collected from water buffaloes. IFAT showed that BoTRAP1 is mainly localized on the apical end of intracellular parasites by using polyclonal antibodies (PcAb) against rBoTRAP1. Meanwhile, the PcAb test also identified the native BoTRAP1 as a $\sim 65 \mathrm{kDa}$ band from $B$. orientalis lysates. The predicted 3D structure of BoTRAP1 contains a metalion-dependent adhesion site (MIDAS), which could be important for interaction with ligand on the surface of the host cells.

Conclusions: Like all known protozoa, B. orientalis has a TRAP family, comprising TRAP1, TRAP2, TRAP3 and TRAP4. The newly identified and characterized BoTRAP1 may play a key role in the invasion of $B$. orientalis into water buffalo erythrocytes.
\end{abstract}

Keywords: Babesia orientalis, Thrombospondin-related anonymous protein 1, Microneme protein, Babesiosis

\footnotetext{
* Correspondence: zhaojunlong@mail.hzau.edu.cn

'State Key Laboratory of Agricultural Microbiology, College of Veterinary

Medicine, Huazhong Agricultural University, Wuhan 430070, Hubei, China

${ }^{2}$ Key Laboratory of Preventive Veterinary Medicine in Hubei Province, Wuhan

430070, Hubei, China

Full list of author information is available at the end of the article
}

(c) The Author(s). 2018 Open Access This article is distributed under the terms of the Creative Commons Attribution 4.0 International License (http://creativecommons.org/licenses/by/4.0/), which permits unrestricted use, distribution, and reproduction in any medium, provided you give appropriate credit to the original author(s) and the source, provide a link to the Creative Commons license, and indicate if changes were made. The Creative Commons Public Domain Dedication waiver (http://creativecommons.org/publicdomain/zero/1.0/) applies to the data made available in this article, unless otherwise stated. 


\section{Introduction}

Babesia orientalis is an apicomplexan parasite that is widespread in southern China and causes babesiosis in water buffaloes, leading to an enormous economic loss $[1,2]$. The clinical symptoms in water buffalo include anemia, fever, icterus, hemoglobinuria and even death [2, 3]. Currently, no vaccine is available to control $B$. orientalis infection, and drugs for treating $B$. orientalis are also scarce, suggesting the importance and necessity to explore potential vaccines based on related antigen molecules.

All the thrombospondin-related anonymous protein (TRAP) family members are secreted by micronemes as a membrane protein, and TRAPs with conserved structures are present in all protozoans, with one or more von Willebrand factor A (vWFA) and thrombospondin type-1 repeat (TSR) domain in their extracellular region, as well as a cytoplasmic tail domain (CTD) with a tryptophan residue [4]. In malaria parasites, the TRAPs were first identified in Plasmodium falciparum, and their homologues were found in all other Plasmodium species $[5,6]$. Subsequent studies have shown that the TRAPs are expressed in different plasmodial stages, such as sporozoite, merozoite and ookinete, and their orthologues are also present in other protozoa, including Toxoplasma gondii, Neospora caninum, Babesia spp., Cryptosporidium spp. and Eimeria spp. [7, 8]. In B. bovis and $B$. gibsoni, the TRAPs were only expressed during the asexual stage, and the antibodies of their recombinant proteins have an obvious influence on Babesia invasion into the host red blood cells (RBCs) $[9,10]$.

In the life-cycle of apicomplexan parasites, host cell invasion is a crucial step for survival, and the process is highly dependent on the interaction between the parasite- and host-surface molecules [11]. In Plasmodium spp., the first step in the invasion of the extracellular merozoites is the attachment to the host cells. In this process, the initial adhesion with host cells based on glycosyl phosphatidylinositol anchor (GPI) of merozoite surface proteins (MSPs) is invertible, followed by reorientation to link the anterior tip of merozoites with the plasma membrane of host cells, leading to the formation of tight junctions from higher-affinity transmembrane proteins secreted by micronemes and rhoptries of parasites; this attachment to the surface of host cells is irreversible. Finally, the parasites invade host cells via a moving complex that involves both apical membrane antigen 1 (AMA1) and rhoptry neck proteins (RONs); this motor process is driven by an actomyosin motor [12]. During the invasion, TRAPs play an important role in the formation of actomyosin motor by linking to actin through their cytoplasmic tail domains (CTD) while binding to host cells via their vWFA domains [7, 13]. Subsequent studies have demonstrated that the interaction between TRAP CTD and actin-myosin is connected by aldolase and depends on the sub-terminal tryptophan residue of cytoplasmic tail [14].

Currently, vaccine development efforts have shifted toward the use of antigenically defined immunogens, particularly the molecules interacting or disrupting the process of parasite invasion into host RBCs [10, 15-17]. Therefore, identification and characterization of these genes encoding TRAPs in Babesia spp. would facilitate the discovery of novel Babesia vaccine candidate antigens.

\section{Methods}

Parasites

Babesia orientalis (Wuhan strain) was isolated from Wuhan city, Hubei Province, China, and preserved in liquid nitrogen with the additive of dimethyl sulfoxide (DMSO) in the State Key Laboratory of Agricultural Microbiology, Huazhong Agricultural University, China.

Two water buffaloes (1.5 years-old) were purchased from a Babesia-free area, and were confirmed to be free of Babesia and Theileria by microscope examination and real-time PCR [18]. The water buffalos were splenectomized two weeks before injection of $4 \mathrm{ml}$ of $B$. orientalis infected blood with the percentage of parasitized erythrocytes (PPE) being 1\%. Blood samples were collected every day to monitor the parasitemia until reaching $3 \%$.

\section{Preparation of RNA and cDNA}

Blood from the jugular vein of experimentally infected water buffaloes was collected in $50 \mathrm{ml}$ centrifuge tubes containing EDTA-K E $_{2}$ (Sigma, Shanghai, China). Total RNA was extracted from purified $B$. orientalis merozoites by using the TransZol Up (TransGen Biotech, Beijing, China) and treated with RNase-free DNaseI (TAKARA, Dalian, China). The cDNA was prepared from $1 \mu \mathrm{g}$ of the total RNA using PrimeScript ${ }^{\text {TM }}$ RT reagent Kit with gDNA eraser (TAKARA, Dalian, China) according to the manufacturer's instructions.

\section{Preparation of recombinant plasmid}

Primer pairs for the full-length and partial BoTRAP1 sequences including a vWFA region and a TSP-1 domain were designed based on the fragment of BoTRAP1 screened from $B$. orientalis genome database (Table 1 ). The PCR reaction was performed using the following cycling parameters: $94{ }^{\circ} \mathrm{C}$ for $5 \mathrm{~min}$, followed by $35 \mathrm{cy}$ cles $\left(94^{\circ} \mathrm{C}\right.$ for $30 \mathrm{~s}, 58{ }^{\circ} \mathrm{C}$ for $30 \mathrm{~s}, 68^{\circ} \mathrm{C}$ for $1 \mathrm{~min}$ ), and a final extension at $68^{\circ} \mathrm{C}$ for $10 \mathrm{~min}$. The resulting PCR product was purified by using EasyPure ${ }^{\odot}$ PCR Purification Kit (TransGEN, Beijing, China), and then cloned into a pET-42b expression vector. All the constructs were confirmed by DNA sequencing. 
Table 1 Oligonucleotide primers used for the amplification of the full-length and partial BoTRAP1 genes

\begin{tabular}{lll}
\hline Primer & Sequence (5'-3') & Restriction enzyme \\
\hline TRAP1-F (full) & ATGATTGGTTACAACAAAATTTGGGCTACG & Spel \\
TRAP1-R (full) & TTAGGCTGCTTCACCCCAAATGTTATTGTC & Xhol \\
TRAP1-F (partial) & TCACTAGTGAAACCTGTATTTCAGGGC (TEV protease site) CTCGACTTCTCCATCGTGG & TRAP1-R (partial) \\
\hline
\end{tabular}

\section{Protein expression and purification}

The recombinant plasmid was transformed into $E$. coli BL21 expression host cells, followed by incubation at $37{ }^{\circ} \mathrm{C}$ in LB medium containing $100 \mathrm{mg} / \mathrm{ml}$ kanamycin for $3 \mathrm{~h}$. At an optical density of 0.6 to 0.8 at $600 \mathrm{~nm}\left(\mathrm{OD}_{600}\right)$, the cells were induced with $0.8 \mathrm{mM}$ IPTG (Biosharp, Anhui, China), further cultured at $25^{\circ} \mathrm{C}$ for another $12 \mathrm{~h}$ and then harvested.

For protein purification, cells were harvested by centrifugation at $7000 \mathrm{rpm}$ for $10 \mathrm{~min}$ in a high-speed refrigerated centrifuge (Hitachi, Tokyo, Japan), resuspended in PBS (pH 7.5) and lysed by passing through a high-pressure homogenizer at 1000 Bar. After centrifugation at $10,000 \times \mathrm{rpm}$ and $4{ }^{\circ} \mathrm{C}$ for $10 \mathrm{~min}$, the supernatant was filtered through a $0.45 \mu \mathrm{m}$ pore size filter and loaded onto glutathione sepharose beads (GE Healthcare, Uppsala, Sweden). The proteins were eluted with

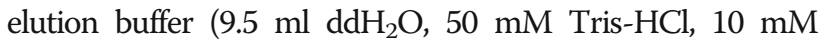
L-glutathione reduced glutathione). The GST-tag on the $\mathrm{N}$-terminus was cleaved using TEV (Tobacco Etch Virus) protease (Solarbio, Shanghai, China) at $4{ }^{\circ} \mathrm{C}$ for $8 \mathrm{~h}$.

\section{Preparation of anti-rBoTRAP1 immune serum}

Polyclonal antibodies against BoTRAP1 protein were prepared in Japanese white rabbits $(n=2)$ according to the established immune procedure. Briefly, seven days after purchase, the rabbits were subcutaneously immunized with the purified $500 \mu \mathrm{g}$ recombinant protein emulsified in equal amounts of Freund's complete adjuvant (Sigma, Shanghai, China), followed by a second immunization with one half of the same recombinant protein emulsified in equal amounts of Freund's incomplete adjuvant (Sigma, Shanghai, China) at a 14-day interval. This was followed by another three immunizations each at 7-day intervals. After the titer of the antisera was assayed by ELISA (the recombinant protein was coated), blood samples were collected from the carotid artery of the rabbits to prepare the polyclonal antibodies. Total immunoglobulin Gs (IgGs) were purified from rabbit sera through a Protein A chromatography column according to the manufacturer's instructions (Beyotime Biotechnology, Shanghai, China).

\section{Identification of immunogenicity and native BoTRAP 1 by Western blot}

The $B$. orientalis-infected RBCs $(1 \mathrm{ml})$ were suspended in an equal amount of phosphate-buffered saline (PBS) buffer, and supplemented with red blood cell lysis buffer preheated at $37^{\circ} \mathrm{C}(18 \mathrm{ml})$, followed by heating in a water bath for $5 \mathrm{~min}$ and centrifugation at 2450× rpm for $5 \mathrm{~min}$. The resulting supernatant was collected and centrifuged at $12,000 \times \mathrm{rpm}$ for $20 \mathrm{~min}$. After a high-speed centrifugation, pellets (parasite fraction) from previous steps were collected and washed three times in cold phosphate-buffered saline (PBS) buffer, followed by the addition of an equal amount of PBS and centrifugation at $15,000 \times \mathrm{rpm}$ for $20 \mathrm{~min}$. After discarding the supernatant, the pellets were suspended in PBS and stored at $-20{ }^{\circ} \mathrm{C}$.

The rBoTRAP1 and $B$. orientalis lysates were separately subjected to $12 \%$ SDS-PAGE using the standard method, followed by electroblotting onto a nitrocellulose membrane. The membranes for the Western blot were blocked with $0.05 \%$ Tween-20 in TBS (TBST) plus $1 \%$ BSA overnight at $4{ }^{\circ} \mathrm{C}$, and then separately probed with the anti-BoTRAP1 PcAb or positive serum of B. orientalis diluted with TBST $(1: 200)$ at $37^{\circ} \mathrm{C}$ for $1 \mathrm{~h}$. The membranes were washed 5 times in TBST and incubated with secondary antibodies diluted with TBST (1:1000, HRP labeled goat anti-rabbit IgG) at $37^{\circ} \mathrm{C}$ for $1 \mathrm{~h}$. After rinsing 5 times in TBST, the positive bands on membranes were visualized using the ECL method.

\section{Localization of BoTRAP1 by indirect florescent antibody test (IFAT)}

The B. orientalis-infected water buffalo RBCs (3\% parasitemia) were smeared on glass slides and fixed in cold $100 \%$ methanol $\left(-20{ }^{\circ} \mathrm{C}\right)$. After three washes, blood smears were permeabilized by using $0.1 \%$ Triton- 100 for $30 \mathrm{~min}$, followed by incubation separately with anti-BoTRAP1 PcAb and pre-immune rabbit sera diluted $200 \times$ with $1 \times$ $\mathrm{PBS} / 1 \% \mathrm{BSA}$ for $30 \mathrm{~min}$. The secondary antibody was anti-rabbit Alexa-594 diluted 1000× with $1 \times$ PBS/1\% BSA and the parasite nucleus was stained with a Hoechst stain. Finally, the coverslips with cells were mounted on a slide in $10 \mu \mathrm{l}$ anti-fluorescence quenching agent and observed with a confocal laser scanning microscope.

\section{Sequence analysis and crystal structure modeling of BoTRAP1}

The BoTRAP1 gene has a significant degree of similarity to the previously reported BbTRAP1 (XM_001609738.1), and was analyzed by Conserved Domain Search Service (CD Search) of NCBI (https://www.ncbi.nlm.nih.gov/Structure/ 
cdd/wrpsb.cgi) for the presence of vWFA and TSP-1 domains. Sequence alignment and phylogenetic analysis of BoTRAP1 with related apicomplexan parasites was performed using the MEGA7 software. The BoTRAP1 3D structure model was generated using SWISS-MODEL (https://swissmodel.expasy.org/interactive), according to the reported crystal structure of Plasmodium vivax TRAP protein (PDB code: 4hqo).

\section{Results}

\section{Characterization of BoTRAP1 gene}

The cDNA sequence of BoTRAP1 had a length 1734 $\mathrm{bp}$, contained five exons and encoded a polypeptide of 577 amino acid residues with a signal peptide and transmembrane region (Fig. 1a). Multiple sequence alignment of the two BoTRAP1 domains with the related TRAP domains of apicomplexan parasites revealed that the two domains from BoTRAP1 have a low similarity, $29 \%, 28 \%, 22 \%, 40 \%, 30 \%$ and $30 \%$, to those of Toxoplasma gondii (GenBank: AAB63303.1), Plasmodium vivax (GenBank: AAC97484), Babesia gibsoni (GenBank: BAI66064.1), Babesia microti (GenBank: XP_012650313.1), Theileria annulata (GenBank: XP_952976.1) and Neospora caninum (GenBank: AAF01565.1), respectively. However, they have a high similarity of $80 \%$ with the two domains of Babesia bovis (GenBank: XP_001609788.1) (Fig. 1b). Phylogenetic analysis based on vWFA and TSP-1 showed that $B$. orientalis has a closer relationship with $B$. bovis than the other apicomplexan parasites (Fig. 1c).

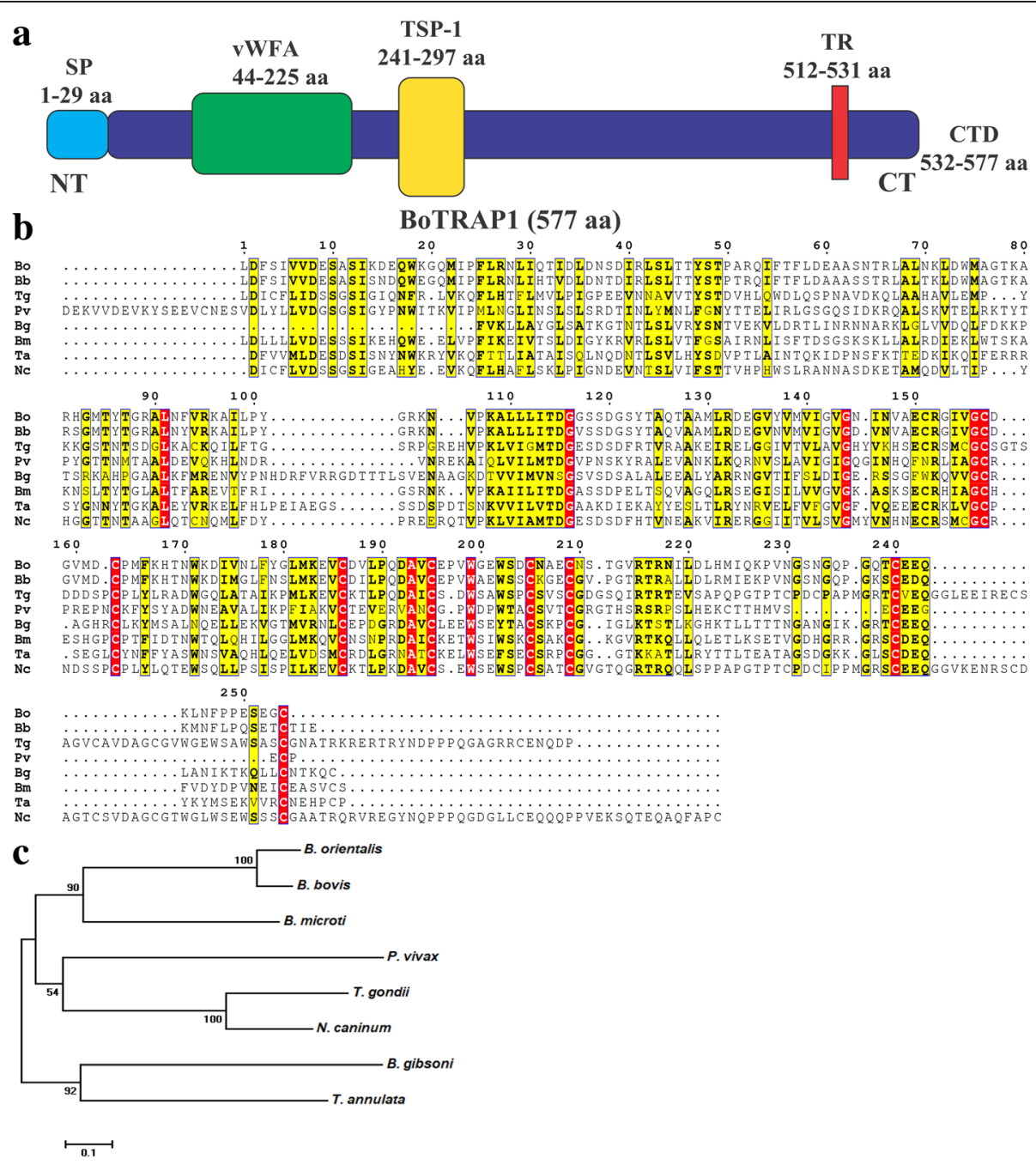

Fig. 1 Alignment of amino acid sequences and phylogenetic analysis of TRAPs. a Graphic depiction of BoTRAP1. A signal peptide at the Nterminus, two functional domains (VWFA and TSP-1), a transmembrane region and a cytoplasmic C-terminal tail domain were dispersed in the coding region. b BOTRAP1 sequence alignment across various strains of apicomplexan parasites, and the sequences were aligned using ClustalW. c Neighbor-joining tree showing phylogenetic relationship of the BoTRAP1 sequences of two conserved regions identified in this study, with sequences of other apicomplexan parasite. The scale-bar represents the nucleotide substitutions per position. Branch lengths represent the amount of genetic distance change between the strains. Abbreviations: SP, signal peptide; TR, transmembrane region; CTD, C-terminal tail domain 


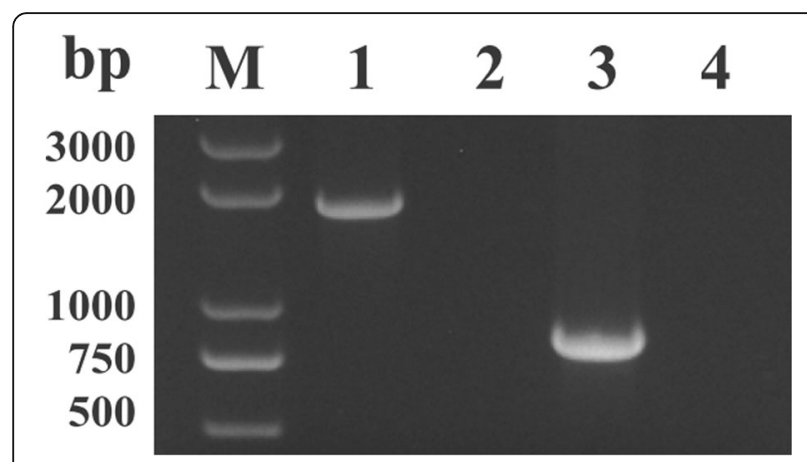

Fig. 2 PCR results of amplifying the full-length and the partial sequence of BOTRAP1 from B. orientalis CDNA. Lane M: molecular weight marker; Lane 1: the full-length BoTRAP1 (1734 bp); Lane 2: negative control; Lane 3: the partial BOTRAP1 including VWFA and TSP-1 domains (762 bp); Lane 4: negative control

\section{Expression of the recombinant BoTRAPI}

The full and partial BoTRAP1 sequences were obtained separately from $B$. orientalis cDNA by PCR (Fig. 2). For molecular characterization of BoTRAP1, the truncated fragment was expressed in $E$. coli with a TEV protease site, and the size of rBoTRAP1 was $\sim 55 \mathrm{kDa}$ including a $26 \mathrm{kDa}$ GST-tag (Fig. 3, Lane 1). The rBoTRAP1 was purified by glutathione sepharose beads (Fig. 3, Lane 4), and the GST tag on the N-terminus was removed by using the TEV protease (Fig. 3, Lane 5).

\section{Immune reactivity of rBoTRAP1 and identification of native BoTRAP1}

The potential of BoTRAP1 as vaccine candidate antigen for preventing and controlling $B$. orientalis infection in water buffalo was tested by Western blot, and two bands corresponding to $55 \mathrm{kDa}$ (GST fusion) and $28 \mathrm{kDa}$ (GST removed) rBoTRAP1 were detected by using positive serum in contrast to no signal observed with negative sera used as control (Fig. 4a). The results indicated that rBoTRAP1 could differentiate $B$. orientalis positive and negative serum samples from water buffalo. To identify native BoTRAP1 in $B$. orientalis, anti-rBoTRAP1 immune sera were produced in rabbits, purified with a Protein A chromatography column, and used to detect $B$. orientalis lysates by Western blot. One band corresponding to $\sim 65 \mathrm{kDa}$ native BoTRAP1 was detected (Fig. 4b), and no signal was found in uninfected water buffalo RBCs and pre-immune sera (data not shown). For further characterization of BoTRAP1, we performed an IFAT separately using anti-BoTRAP1 rabbit immune serum and pre-immune sera. The results showed that BoTRAP1 is localized on the apical end of intracellular or extracellular parasite under a confocal laser scanning microscope (Fig. 5a). No signals were observed in parasites when tested using pre-immune sera (Fig. 5b).

\section{Homology modeling of BoTRAP1}

To further confirm the potential role of BoTRAP1 in the invasion of parasites into host cells, the 3D structures of BoTRAP1 were homology-modeled using the crystal structure of $P$. vivax TRAP protein as a template. As shown in Fig. $6 \mathrm{~b}$, the predicted BoTRAP1 3D model contain vWFA and TSP-1 domains, with an inserted domain in the integrin of the vWFA domain, containing a metalion-dependent adhesion site (MIDAS) for interaction with ligand. While the amino acid sequences are not well conserved between

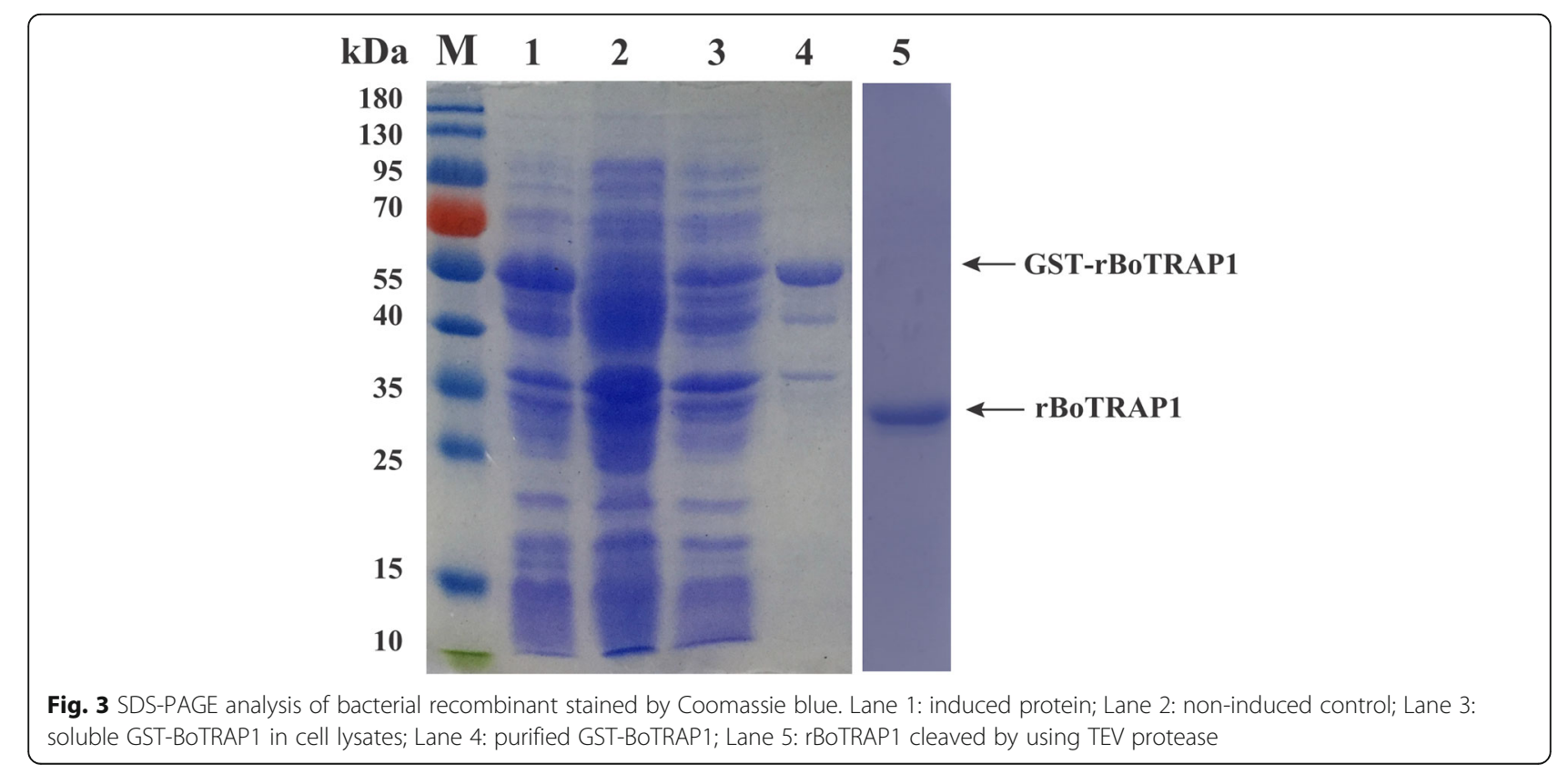


a

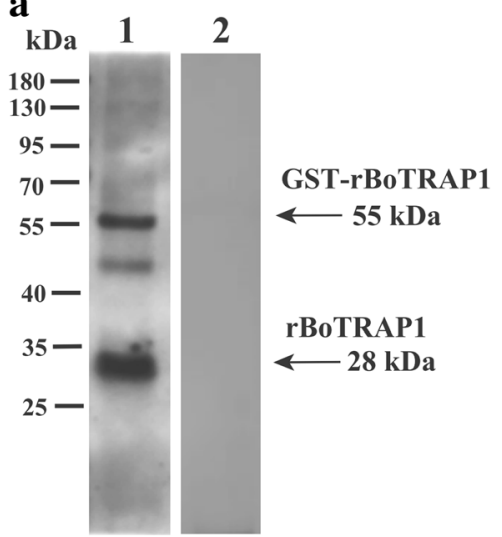

b

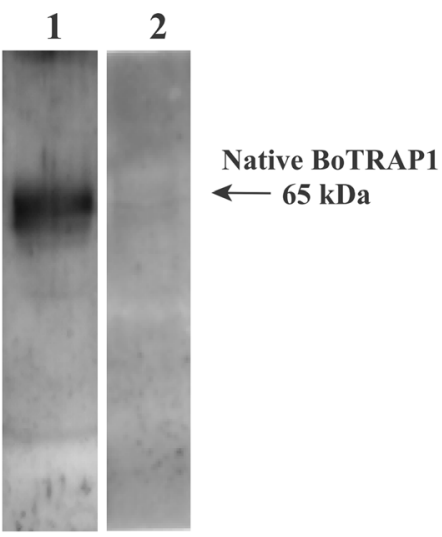

Fig. 4 Western blot analysis of BoTRAP1. a Determination of antibody response of rBoTRAP1 in water buffalo antiserum. Lane M: molecular weight marker; Lane 1: rBoTRAP1 reacted with B. orientalis positive serum; Lane 2: rBoTRAP1 probed with B. orentalis non-infected serum from water buffalo. b Determination of native BoTRAP1 in the merozoite stage. B. orientalis lysates (Lane 1) and non-infected bovine RBCs lysates (Lane 2) were probed with anti-BoTRAP1 rabbit serum

BoTRAP1 and PvTRAP (28\% similarity), their overall structures share a conserved feature (Fig. 6a, b).

\section{Discussion}

Four TRAP genes have been identified in $B$. bovis and named BbTRAP1-4 (GenBank accession numbers XM_001609738, XM_001609762, XM_001609736 and XM_001609760, respectively). The BbTRAPs share a typical structure with other apicomplexan TRAPs, including vWFA and TSP domains as well as a transmembrane region followed by a cytoplasmic Cterminal tail domain, except for the absence of the TSP domain from BbTRAP4 [19]. The TRAP family has also been identified in $B$. orientalis and named as TRAP1-4 (Data for TRAP2-4 not shown). Sequence analysis indicated that the four TRAP genes are very similar in both B. bovis and B. orientalis.

The TRAPs are secreted from micronemes as a transmembrane protein and are present in all apicomplexan parasites, with a high similarity for their cellular localization in Plasmodium spp., Neospora caninum, B. bovis and $B$.

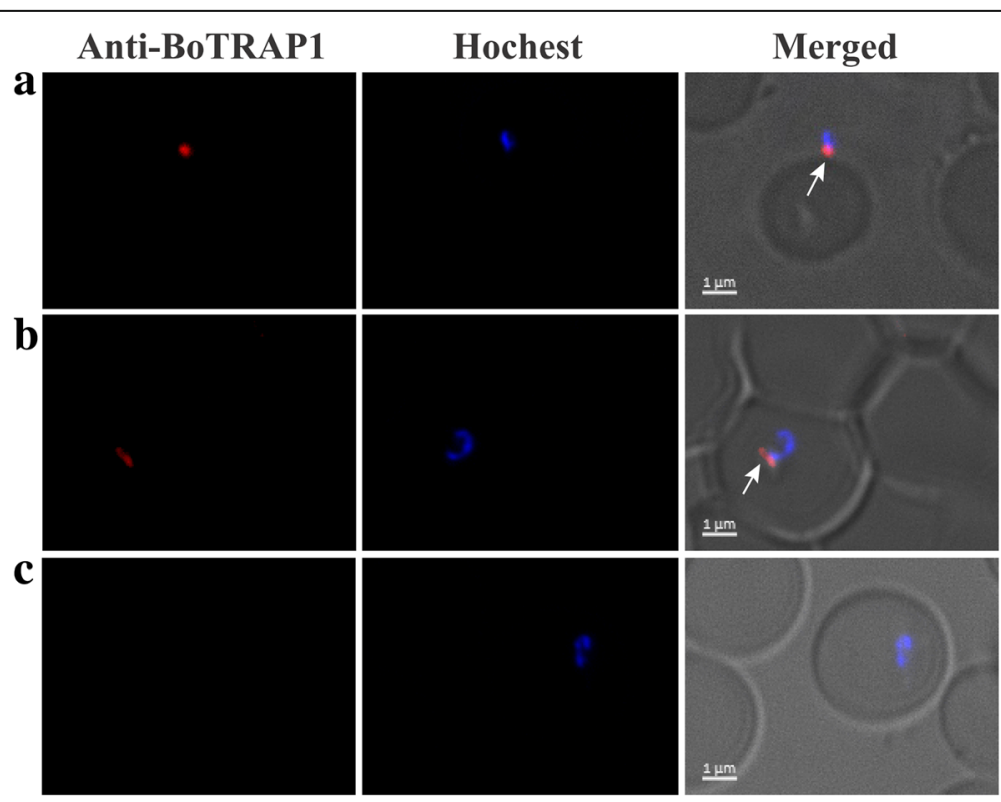

Fig. 5 Localization of BOTRAP1 on B. orientalis by immunofluorescence analysis. Babesia orentalis-infected RBCs were stained separately with PCAb-BOTRAP1 and pre-immune serum. Reactivity of the anti-rBoTRAP1 serum with extracellular parasite (a) and intracellular parasite single (b). Pre-immune sera were used as negative control for the validation test (c). PcAb-BoTRAP1 (Red) reacted with native TRAP1 on merozoites. Nuclei were counterstained by Hochest (blue). Scale-bars: $1 \mu \mathrm{m}$ 

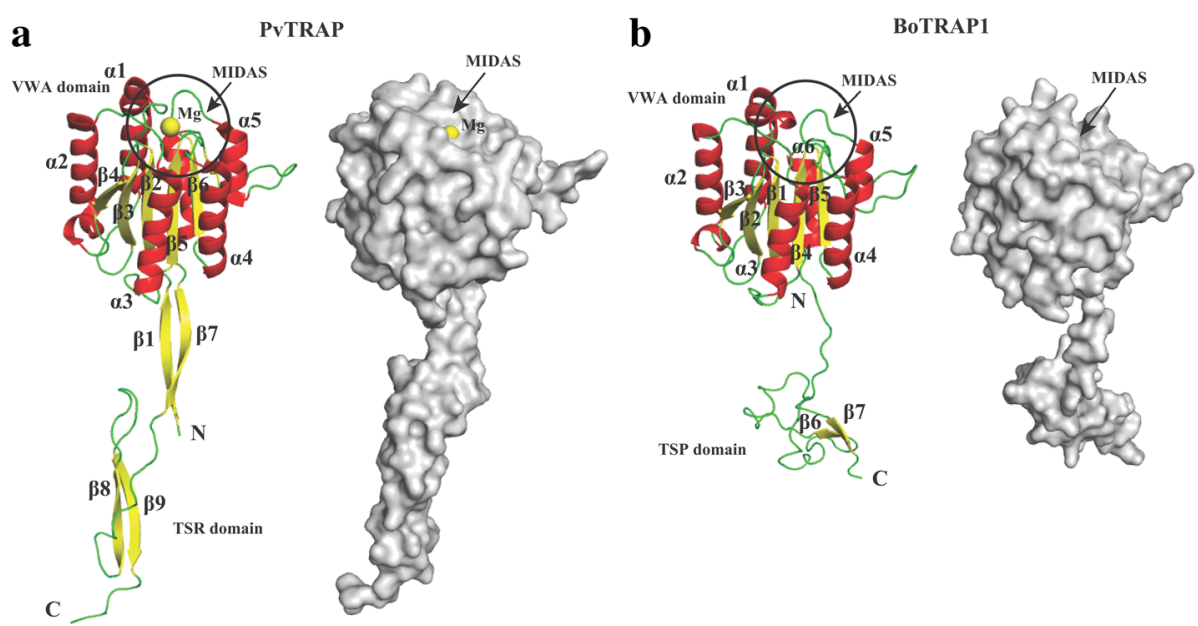

Fig. 6 3D structure model of BOTRAP1 constructed by using SWISS-MODEL. a Crystal structure of PVTRAP (residues 25-283). The structure of PVTRAP is shown as a cartoon (left) and surface structure (right). The $\mathrm{Mg}^{2+}$ ion at the ligand binding site was colored in yellow. The a-helices were shown as red, and yellow indicates $\beta$-strands. b Predicted BoTRAP1 structure (residues 44-297). The 3D structure in identical orientations contains six a-helices (a1 and a6) and seven $\beta$-strands ( $\beta 1$ to $\beta 7$ ). The BoTRAP1 VWFA domain also includes a metalion-dependent adhesion site (MIDAS) for binding ligand. The figure on the left shows the cartoon structure of BoTRAP1, and the figure on the right shows BoTRAP1 surface structure

gibsoni [7, 10, 19, 20]. Confocal laser microscopy revealed that the native BoTRAP1 is located in the apical end of parasites, which is consistent with the TRAP localization of other parasites. On Western blot, the anti-rBoTRAP1 antibody detected the native protein as a $\sim 65 \mathrm{kDa}$ band from $B$. orientalis lysates. This protein size is in agreement with the theoretical mass of native BoTRAP1 (65 kDa) and is approximately the size of BbTRAP1 $(73 \mathrm{kDa})$. Together, these results further verified that the sequence we obtained from $B$. orientalis CDNA precisely is the BoTRAP1 gene.

Furthermore, we tried to determine the ectodomain crystal structure of BoTRAP1 (vWFA and TSP-1 domains). Despite a high purity and high concentration, the rBoTRAP1 failed to grow crystals in crystal buffer (576-well) (Hampton, California, USA). This result is probably attributed to the use of the prokaryotic, rather than the eukaryotic, expression system, leading to the absence of glycosylation on the surface of the recombinant protein. Fortunately, the overall structure of TRAP family is quite conservative across all apicomplexan parasites [21, 22].
For further characterization, the BoTRAP1 ectodomain structure was simulated by homologous modeling.

The interaction between the parasite ligands and the corresponding host-cell receptors is essential to the invasion of apicomplexan parasites [4, 7]. Currently, the invasion mechanism of apicomplexan parasites is poorly understood. A related study has shown that TRAPs participate in the formation of actin-myosin motor and drive the parasites into the host cells, enabling the translocation of TRAPs to the posterior pole of the parasites along the cytomembrane surface [23]. However, the actin-myosin motor was mediated by a sub-terminal tryptophan residue within the cytoplasmic tail of TRAPs, and the tryptophan residue connected to aldolase is rather conserved across apicomplexan parasites [4]. Interestingly, in order to release the parasites into host cells completely, the TRAPs translocated to the posterior end from the apical end of parasites need to be cleaved at their transmembrane domains by the rhomboid proteases (ROMs) [24, 25]. In this study, we predicted the

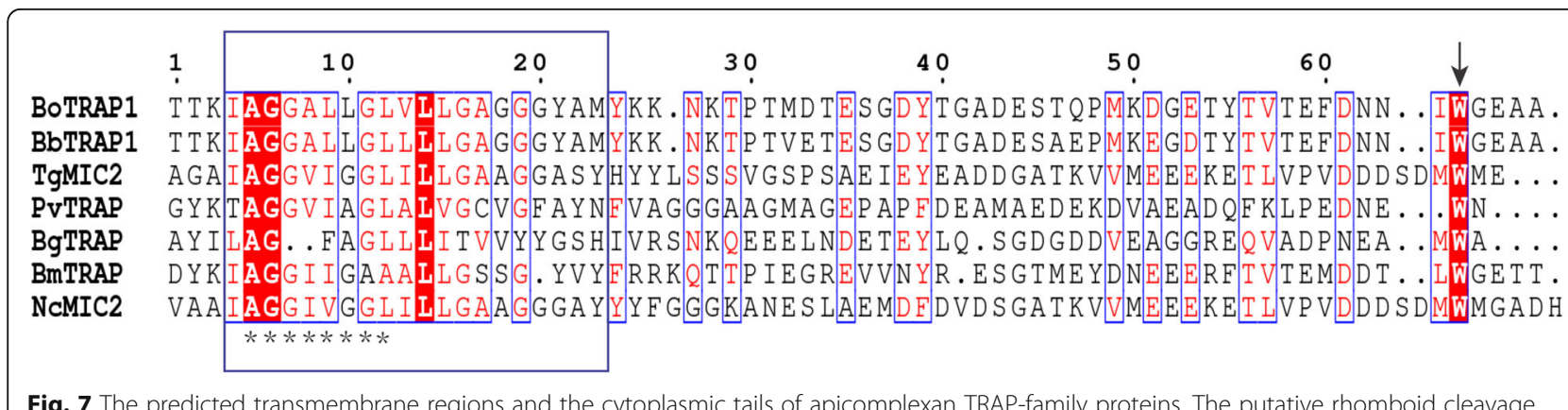

sites are marked with asterisks, the predicted transmembrane domains by rectangles, and the sub-terminal tryptophan residues with an arrow 
transmembrane region and the putative rhomboid cleavage site of BoTRAP1, as well as detected the conserved tryptophan residue present, in the cytoplasmic tail domain (Fig. 7).

In malarial parasites, the GPI-linked protein semaphorin7A (CD108) had been identified as an erythrocyte surface receptor for the P. falciparum MTRAP [26]. However, the erythrocyte receptor is still unknown for the BoTRAPs. Therefore, exploring the interaction of BoTRAPs with erythrocyte surface would facilitate the understanding of the invasion mechanism of $B$. orientalis and the discovery of new blocking agents or methods for controlling $B$. orientalis.

\section{Conclusions}

Overall, BoTRAP1 was cloned, sequenced and expressed, with the native BoTRAP1 being characterized as $\sim 65 \mathrm{kDa}$ by Western blot and located in the apical end of the parasites. The simulation structure and functional analysis revealed that BoTRAP1 might perform critical functions in aiding parasite invasion and interaction of parasite-host RBCs. Blocking TRAP1 function might prevent the parasites from invading RBCs. Therefore, the newly identified and characterized BoTRAP1 may have potential as drug target or vaccine candidate antigen for preventing and controlling B. orientalis infection in water buffalo.

\section{Abbreviations}

TRAP: thrombospondin-related anonymous protein; vWFA: Von Willebrand factor A; TSR: Thrombospondin type-1 repeat; CTD: Cytoplasmic tail domain; MIDAS: Metalion-dependent adhesion site; TEV: Tobacco Etch Virus; GPI: Glycosyl phosphatidylinositol anchor; PPE: Percentage of parasitized erythrocytes; PBS: Phosphate-buffered saline; DMSO: Dimethylsulfoxide; ROMs: Rhomboid proteases; RBC: Red blood cell; BSA: Bull serum albumin

\section{Acknowledgements}

Not applicable.

\section{Funding}

This work was supported by the National Key Basic Research Program (973 Program) of China (2015CB150302), the National Natural Science Foundation of China (31772729), the National Key Research and Development Program of China (2017YFD0501201) and the Natural Science Foundation of Hubei Province (2017CFA020).

\section{Availability of data and materials}

The nucleotide sequence generated during this study was submitted to the GenBank database under the accession number MH715938.

\section{Authors' contributions}

$L Y, L H$ and JZ designed the study and drafted the manuscript. QL, XZ, YH, $Y S, Z N, Y Z, X A, M L, S W, Y A$ and $C H$ performed the experiments and analyzed the results. All authors have read and approved the final manuscript.

\section{Ethics approval}

All the experimental animals were housed and treated in accordance with the stipulated rules for the Regulation of the Administration of Affairs Concerning Experimental Animals of PR China, Laboratory Animals Research Centre of Hubei province and the Ethics Committee of Huazhong Agricultural University (permit number HZAUCA-2017-005).

\section{Consent for publication}

Not applicable.

\section{Competing interests}

The authors declare that they have no competing interests.

\section{Publisher's Note}

Springer Nature remains neutral with regard to jurisdictional claims in published maps and institutional affiliations.

\section{Author details}

${ }^{1}$ State Key Laboratory of Agricultural Microbiology, College of Veterinary Medicine, Huazhong Agricultural University, Wuhan 430070, Hubei, China. ${ }^{2}$ Key Laboratory of Preventive Veterinary Medicine in Hubei Province, Wuhan 430070, Hubei, China. ${ }^{3}$ College of Life Science, Longyan University, Longyan 364012, Fujian, China. ${ }^{4}$ Fujian, Provincial Key Laboratory for the Prevention and Control of Animal Infectious Diseases and Biotechnology, Longyan 364012, Fujian, China. ${ }^{5}$ Key Laboratory of Animal Epidemical Disease and Infectious Zoonoses, Ministry of Agriculture, Huazhong Agricultural University, Wuhan 430070, Hubei, China.

Received: 30 August 2018 Accepted: 29 November 2018

Published online: 27 December 2018

\section{References}

1. Yao B, Zhao J, Liu E, Ding S, Shi J, Liu Z. Serological investigations on Babesia orientalis infection. Status of water buffaloes in Hubei Province. Parasitol Res. 2002;88:S11-2.

2. Liu Z, Zhao J, Ma L, Yao B. Studies on buffalo babesiosis in Hubei Province, China. Trop Anim Health Prod. 1997;29:33S-6S.

3. Liu Q, Zhao J, Zhou Y, Liu E, Yao B, Fu Y. Study on some molecular characterization of Babesia orientalis. Vet Parasitol. 2005;130:191-8.

4. Morahan BJ, Wang L, Coppel RL. No TRAP, no invasion. Trends Parasitol. 2009:25:77-84.

5. Robson KJ, Dolo A, Hackford IR, Doumbo O, Richards MB, Keita MM, et al. Natural polymorphism in the thrombospondin-related adhesive protein of Plasmodium falciparum. Am J Trop Med Hyg. 1998;58:81-9.

6. Templeton TJ, Kaslow DC. Cloning and cross-species comparison of the thrombospondin-related anonymous protein (TRAP) gene from Plasmodium knowlesi, Plasmodium vivax and Plasmodium gallinaceum. Mol Biochem Parasitol. 1997:84:13-24.

7. Baum J, Richard D, Healer J, Rug M, Krnajski Z, Gilberger TW, et al. A conserved molecular motor drives cell invasion and gliding motility across malaria life cycle stages and other apicomplexan parasites. J Biol Chem. 2006;281:5197-208.

8. Baum J, Gilberger TW, Frischknecht F, Meissner M. Host-cell invasion by malaria parasites: insights from Plasmodium and Toxoplasma. Trends Parasitol. 2008;24:557-63.

9. Gaffar FR, Yatsuda AP, Franssen FF, de Vries E. A Babesia bovis merozoite protein with a domain architecture highly similar to the thrombospondinrelated anonymous protein (TRAP) present in Plasmodium sporozoites. Mol Biochem Parasitol. 2004;136:25-34.

10. Zhou J, Fukumoto S, Jia H, Yokoyama N, Zhang G, Fujisaki K, et al. Characterization of the Babesia gibsoni P18 as a homologue of thrombospondin related adhesive protein. Mol Biochem Parasitol. 2006;148: 190-8.

11. Sibley LD. Intracellular parasite invasion strategies. Science. 2004;304:248-53.

12. Soldati-Favre D. Molecular dissection of host cell invasion by the apicomplexans: the glideosome. Parasite. 2008;15:197-205.

13. Jones ML, Kitson EL, Rayner JC. Plasmodium falciparum erythrocyte invasion: a conserved myosin associated complex. Mol Biochem Parasitol. 2006;147: 74-84.

14. Buscaglia CA, Coppens I, Hol WG, Nussenzweig V. Sites of interaction between aldolase and thrombospondin-related anonymous protein in Plasmodium. Mol Biol Cell. 2003;14:4947-57.

15. Fish $L$, Leibovich B, Krigel $Y$, McElwain T, Shkap V. Vaccination of cattle against $B$. bovis infection with live attenuated parasites and non-viable immunogens. Vaccine. 2008;26:G29-33.

16. Morgan RE, Evans KM, Patterson S, Catti F, Ward GE, Westwood NJ. Targeting invasion and egress: from tools to drugs? Curr Drug Targets. 2007; 8:61-74.

17. Nemetski SM, Cardozo TJ, Bosch G, Weltzer R, O'Malley K, Ejigiri I, et al. Inhibition by stabilization: targeting the Plasmodium falciparum aldolaseTRAP complex. Malar J. 2015;14:324 
18. He L, Feng H, Zhang Q, Zhang W, Khan MK, Hu M, et al. Development and evaluation of real-time PCR assay for the detection of Babesia orientalis in water buffalo (Bubalus bubalis Linnaeus, 1758). J Parasitol. 2011;97:1166-9.

19. Terkawi MA, Ratthanophart J, Salama A, AbouLaila M, Asada M, Ueno A, et al. Molecular characterization of a new Babesia bovis thrombospondinrelated anonymous protein (BbTRAP2). PLoS One. 2013;8:e83305.

20. Lovett JL, Howe DK, Sibley LD. Molecular characterization of a thrombospondin-related anonymous protein homologue in Neospora caninum. Mol Biochem Parasitol. 2000;107:33-43.

21. Song G, Koksal AC, Lu C, Springer TA. Shape change in the receptor for gliding motility in Plasmodium sporozoites. Proc Natl Acad Sci USA. 2012; 109:21420-5.

22. Song G, Springer TA. Structures of the Toxoplasma gliding motility adhesin. Proc Natl Acad Sci USA. 2014;111:4862-7.

23. Menard R. The journey of the malaria sporozoite through its hosts: two parasite proteins lead the way. Microbes Infect. 2000;2:633-42.

24. Baker RP, Wijetilaka R, Urban S. Two Plasmodium rhomboid proteases preferentially cleave different adhesins implicated in all invasive stages of malaria. PLoS Pathog. 2006;2:e113.

25. Brossier F, Jewett TJ, Sibley LD, Urban S. A spatially localized rhomboid protease cleaves cell surface adhesins essential for invasion by Toxoplasma. Proc Natl Acad Sci USA. 2005:102:4146-51.

26. Bartholdson SJ, Bustamante LY, Crosnier C, Johnson S, Lea S, Rayner JC, et al. Semaphorin-7A is an erythrocyte receptor for $P$. falciparum merozoitespecific TRAP homolog, MTRAP. PLoS Pathog. 2012;8:e1003031.

Ready to submit your research? Choose BMC and benefit from:

- fast, convenient online submission

- thorough peer review by experienced researchers in your field

- rapid publication on acceptance

- support for research data, including large and complex data types

- gold Open Access which fosters wider collaboration and increased citations

- maximum visibility for your research: over $100 \mathrm{M}$ website views per year

At $\mathrm{BMC}$, research is always in progress.

Learn more biomedcentral.com/submissions 